\title{
LAS CONSTRUCCIONES AGRARIAS EN LA COMUNIDAD DE MADRID Y SUS CONDICIONANTES ESTÉTICOS SEGÚN LAS NORMAS SUBSIDIARIAS
}

\author{
(AGRICULTURAL BUILDING IN THE COMUNIDAD DE MADRID AND THEIR \\ AESTHETIC DETERMINING FACTORS ACCORDING TO SUBSIDIARY \\ STANDARDS)
}

Ignacio Cañas Guerrero, ingeniero agrónomo

ETSE Agrónomos, Universidad de Santiago de Compostela. Campus de Lugo ESPANA

Fecha de recepción: 26 - IV - 93

\begin{abstract}
RESUMEN
Las construcciones agrarias, por sus caracteristicas, suelen situarse fuera del núcleo y por ello potencialmente pueden degradar los recursos paisajísticos.
\end{abstract}

En la Comunidad de Madrid existe una abundante normativa sobre las modificaciones antrópicas; nos proponemos en este artículo estudiar la legislación que afecte de forma más directa a las construcciones agrarias.

\section{SUMMARY}

Agricultural building are, due to their characteristics, usually located outside the urban village and may therefore spoil the landscape.

In the Comunidad de Madrid there is an extensive standard on the anthropic modifications. In the present article we study the legislation which directly affects agricultural building.

\section{INTRODUCCIÓN}

El estudio de la normativa de los municipios madrileños se ha realizado por considerar que puede servir para:

- Estudiar de forma general cómo está protegido el paisaje en el ámbito de la Comunidad frente a las construcciones.

- Prever en un futuro inmediato la posible evolución de la integración en el paisaje de los edificios.

- Tener una fuente de la valoración del paisaje y de las construcciones por quienes viven en el municipio, a partir de la base que las normas recogen la opinión de los observadores del municipio.

\section{LA PROTECCIÓN DEL PAISAJE ANTE LAS NUEVAS CONSTRUCCIONES EN LA COMUNIDAD AUTÓ- NOMA DE MADRID (CAM)}

Hemos escogido la Comunidad Autónoma de Madrid además de por los motivos técnicos, ya que en Madrid la presión antrópica es grande, por existir una financiación de esta Comunidad. Pensamos no obstante que la situación puede generalizarse a otras comunidades autónomas.

La protección del paisaje por lo que se refiere a las nuevas construcciones en la Comunidad de Madrid se establece de forma genérica en la Ley del Suelo(1) y su desarrollo reglamentario, y así mismo en la ley 8/1990 de 25 de julio sobre Reforma del Régimen Urbanístico y Valoraciones del suelo. Pero es la Comunidad de Madrid, al amparo del artículo 31.1 de la ley del suelo y de acuerdo con el Estatuto de autonomía, la que tiene plenitud de la función legislativa en materia de ordenación del territorio y urbanismo.

La Comunidad Autónoma, en su creciente preocupación por preservar las áreas de valor paisajístico del impacto de las nuevas construcciones, aprobó la Ley sobre Medidas de Disciplina Urbanǐstica de la Comunidad de Madrid (Ley 4/84 de 10-de febrero) y la Ley sobre ordenación territorial de la 


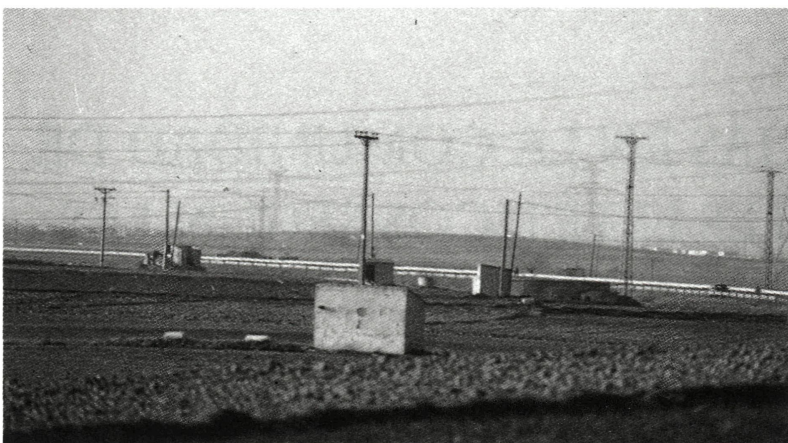

Las instalaciones auxiliares agrarias, es uno de los temas olvidados por la mayoria de las normas. Como se ve en esta fotografía, tomada en el municipio de Torrejón de Velasco, la suma de pequeñas alteraciones puede hacer cambiar totalmente el carácter del paisaje.

Comunidad de Madrid (Ley 10/1984, de 30 de mayo). Además a través de la Consejería de Política Territorial "está exigiendo la formulación de un planeamiento municipal útil... en todos los municipios de la Comunidad de Madrid" (Dirección General de Urbanismo 1990b). Por ello la Consejería de Política Territorial, de acuerdo con los distintos municipios, ha redactado diversas figuras de planeamiento:

- Plan general de ordenación urbana.

- Delimitación del suelo urbano.

- Normas subsidiarias.

El Plan General de Ordenación Urbana requiere una programación: Plan de Etapas y Programa Económico-Financiero que "exige una capacidad de gestión y actuación municipal que desborda ampliamente a municipios de tamaño inferior a los 50.000 habitantes" (Dirección General de Urbanismo 1989). Por ello sólo los grandes municipios como son: Aranjuez, Alcorcón, Getafe, Leganés, Majadahonda, etc., o los que tienen una presión inmobiliaria grande, son los que suelen tener Plan General de Ordenación. No obstante desde el punto de vista que aquí interesa -Normativa de protección del paisaje y condicionamientos estéticos- no existe diferencia entre el Plan General y las Normas Subsidiarias, por ello los consideraremos los dos bajo el epígrafe de Normas Subsidiarias.

CONTENIDO DE LAS NORMAS SUBSIDIARIAS DE PLANEAMIENTO MUNICIPAL DE LA CAM

Las Normas Subsidiarias de Planeamiento Municipal (N.S.P.M) regulan de forma general y para la totali-

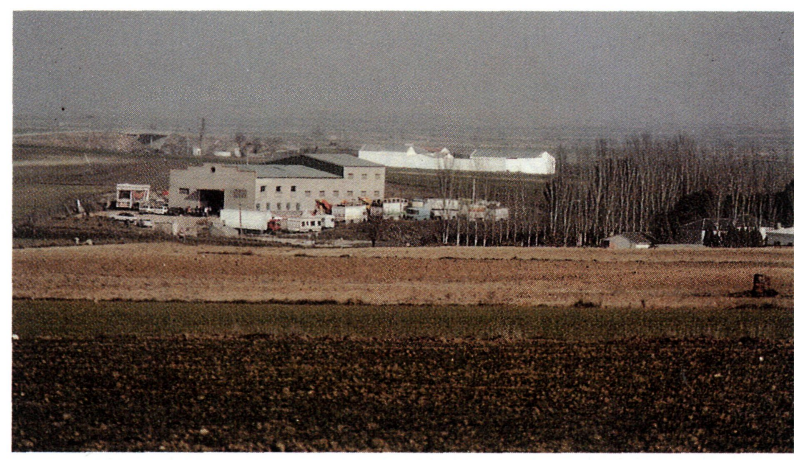

La intensidad o saturación de los colores puede afectar de forma negativa al paisaje. No es suficiente limitar los colores (tintes) sino también su saturación.

dad del término municipal las condiciones de protección del medio-ambiente y el patrimonio social, cultural y económico. Suelen contener los siguientes documentos:

1.- Memoria justificativa de la ordenación

2.- Normas urbanísticas

3.- Catálogo de protección

4.- Planos de ordenación

En el caso del Plan General de Ordenación Urbana, a estos documentos se les añade un programa de actuación y un estudio económico-financiero.

La memoria justificativa de la ordenación es una exposición de los motivos que han llevado a la redacción de las Normas Subsidiarias.

Las normas urbanísticas y los planos de ordenación describen pormenorizadamente las posibles actuaciones de las diversas instalaciones para todo el territorio municipal, teniendo ambos documentos un carácter regulador.

El catálogo de protección tiene por objeto "la protección y conservación de los bienes inmuebles que por sus valores arquitectónicos, urbanísticos, históricos, artísticos, culturales, ambientales, paisajísticos, botánicos, forestales, agrícolas u otros, sean susceptibles de ser considerados como bienes catalogables con arreglo a lo dispuesto en los artículos 12.1.d y 25 de la Ley sobre Régimen del Suelo y Ordenación Urbana" (Dirección General de Urbanismo 1990).

En las normas urbanísticas es dōnde se regulan y dan indicaciones para la integración de la construc- 


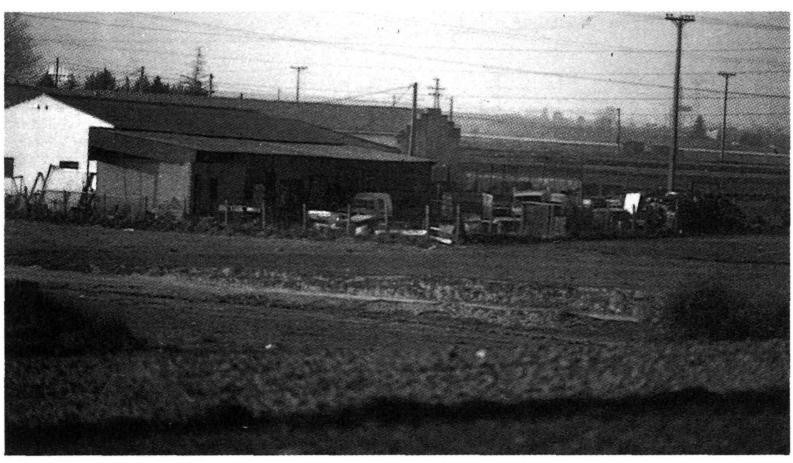

Quizá uno de los grandes problemas que nos encontramos es la falta de sensibilidad por parte de los agricultores. Pensamos que son ellos, principalmente, los que deben conservar y proteger sus recursos visuales evitando que almacenes sin cuidar estén en zonas de alta visibilidad.

ción en el paisaje; este documento consta de dos grandes grupos de normas: normas generales y normas particulares (que a su vez puede ser para el suelo no urbanizable, para el suelo urbano o para el urbanizable $\left.{ }^{(2)}\right)$.

Las normas generales suelen tener los siguientes $\operatorname{apartados}^{(3)}$ :

* Normas de uso del suelo.

* Normas de protección:

- Protección medio ambiental.

- Protección paisajística y de la escena urbana.

- Protección del patrimonio edificado.

* Normas de edificación:

- Condiciones que afectan a las parcelas.

- Condiciones que afectan a las construcciones:

- Condiciones de posición de las construcciones.

- Condiciones de volumen (aprovechamiento).

- Condiciones de la edificación: interiores y exteriores.

* Normas de urbanización:

- Espacio exterior no accesible.

- Espacio exterior accesible:

- Red viaria en suelo urbano y no urbanizable.

- Zonas verdes y espacios libres.

- Equipamientos.

Para el estudio de la integración de las construcciones agrarias en el paisaje los apartados que más directamente les afectan son:

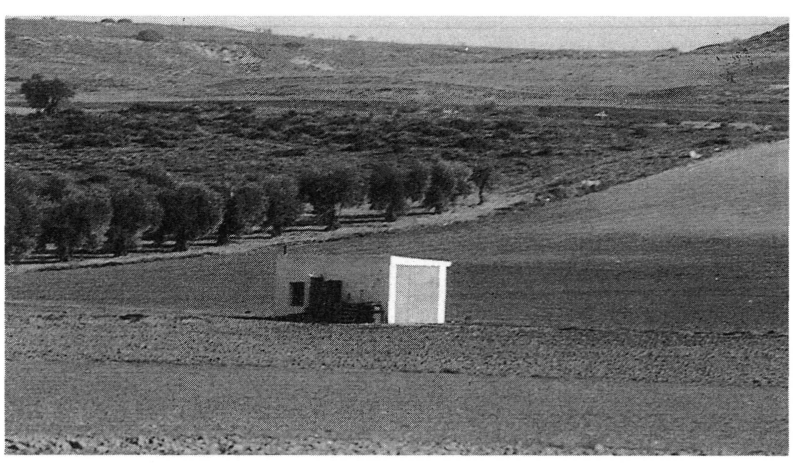

Las normas Subsidiarias o cualquier otra normativa que afecte a suelo no urbanizable debe limitar y regular las formas, colores, número, etc. de las pequeñas instalaciones agrarias.

* Las normas de protección y dentro de éstas las normas de protección paisajística y de la escena urbana, ya que la protección medioambiental se centra en aspectos de vertidos, contaminación acústica y contra incendio.

* Las normas de edificación donde se estudian los siguientes aspéctos:

$1^{\circ}$ Las condiciones de las parcelas suelen determinarse por: la definición de parcela edificable, frente de parcela, fondo de parcela, distancia entre alineaciones, espacios libres privados y públicos y rasantes.

$2^{\circ}$ Las condiciones de posición de las construcciones suelen incluir: el retranqueo de fachada, retranqueo a lindero, línea de fachada, etc.

$3^{\circ}$ Las condiciones de aprovechamiento de las construcciones dentro de la parcela, o condiciones de volumen aquí se estudia entre otras variables: edificabilidad bruta, superficie máxima construible, superficie ocupable, fondo máximo edificable, etc.

$4^{\circ}$ Condiciones constructivas. Las normas suelen llamarle a estas condiciones "Condiciones de forma y buena construcción". Estas incluyen:

$4^{\circ}$ a) Condiciones interiores: iluminación y ventilación, etc, que no se estudiarán.

$4{ }^{\circ}$ b) Condiciones exteriores: tipos edificatorios, altura de la edificación, patios, accesos, cubierta, fachada, cuerpos y elementos salientes y entrantes, carpintería y cerramientos exteriores. 


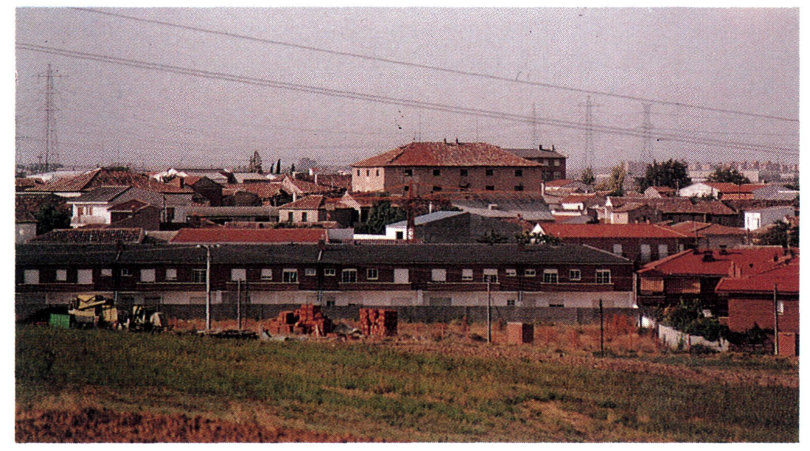

Normalmente en la normativa se suele hacer bastante hincapié en los condicionantes de volumen, olvidándose del resto de condicionantes estéticos como es, en este caso, el color. La importancia de éste es en algunos de nuestros pueblos manifiesta. Pensemos por ejemplo en los pueblos blancos de Andalucía on los colores terrosos de las zonas castellanas.
Dentro de éstas, las condiciones de los puntos 1으, $2^{\circ}, 3^{\circ}$ y $4^{\circ}$ a son más propias de un estudio de urbanismo que objeto de interés para este trabajo. Dentro del punto $4^{\circ} \mathrm{b}$ :

- Los tipos edificatorios están pensados para el núcleo rural, ya que hace referencia sobre todo a alineaciones oficiales, por lo que no los consideraremos.

- Los patios se suelen establecer en función del uso de los locales que abren a ellos, por lo que vuelve a entrar en aspectos urbanísticos.

Por lo tanto los elementos que se estudiaran dentro del epígrafe de las condiciones constructivas son: altura de la edificación, cubierta, fachada, cuerpos y elementos salientes y entrantes, carpintería y cerramientos exteriores.

\section{SELECCIÓN DE LOS MUNICIPIOS DE LA CAM}

Como se trata de estudiar el impacto de las construcciones agrarias se seleccionaron los municipios que Navajas (1983) clasifica como núcleos rurales o villas, desechándose los ruurbanos y urbanos; entre estos grupos se eligieron 40 pueblos (30 núcleos rurales y 10 villas) que representan todas las zonas geográficas en las que se ha dividido la Provincia de Madrid quedándonos los siguientes:

Núcleos rurales serranos:

Sierra Norte: La Acebeda. El Atazar. El Berrueco. Cervera de Buitrago. Gascones. La Hiruela. Horcajo

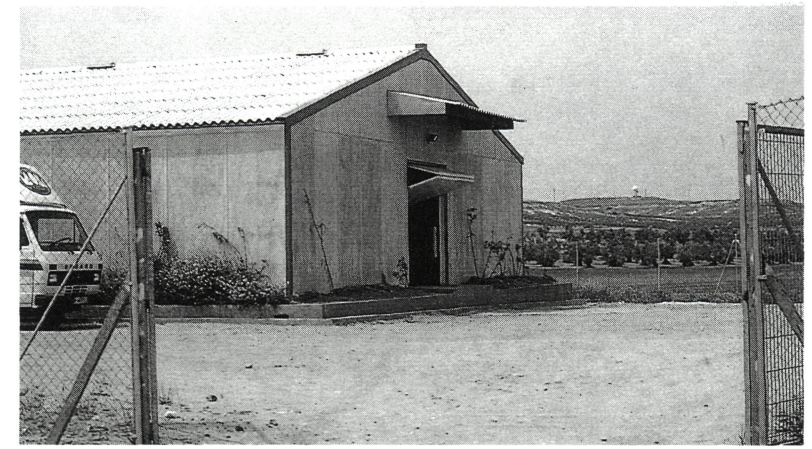

Las texturas brillantes de las cubiertas llaman desafortunadamente la atención. Materiales de chapa metálica deberian estar especialmente probibidos por las diversas normativas. de la Sierra. Montejo de la Sierra. Robregordo. Puebla de la Sierra. La Serna del Monte. Somosierra. Villavieja de Lozoya.

Sierra Centro: Redueña. Santa María de la Alameda. Sierra Suroeste: Las Rozas de Puerto Real.

Núcleos rurales de la meseta:

La Alcarria: Belmonte de Tajo. Brea de Tajo. Campo Real. Estremera. Valdaracete. Villalbilla.

La Sagra: Batres. Casarrubuelos. Villanueva de la Cañada.

El Jarama-Henares: Cobeña. Fresno del Torote. Titulcia. Valdepiélagos. Valdetorres de Jarama.

Villas:

Buitrago de Lozoya. Ciempozuelos. Colmenar de Oreja. Chinchón. Manzanares el Real. Navalcarnero. San Martín de Valdeiglesias, Torrejón de Velasco. Torrelaguna. Valdemoro.

Rurales puros:

Casarrubuelos, El Berrueco, El Atazar, Fresno del Torote, La Hiruela, La Acebeda, Puebla de la Sierra, Santa María de la Alameda.

Dentro de los municipios estudiados podemos hacer dos grandes grupos:

$1^{\circ}$ Grupo de aquellos municipios que tienen editadas las Normas Subsidiarias o plan General de Ordenación Urbana: Batres, Brea del Tajo, Buitrago de Lozoya, Ciempozuelos, Cobeña, Colmenar de Oreja, Chinchón, Estremera, Horcajo de la Sierra, 


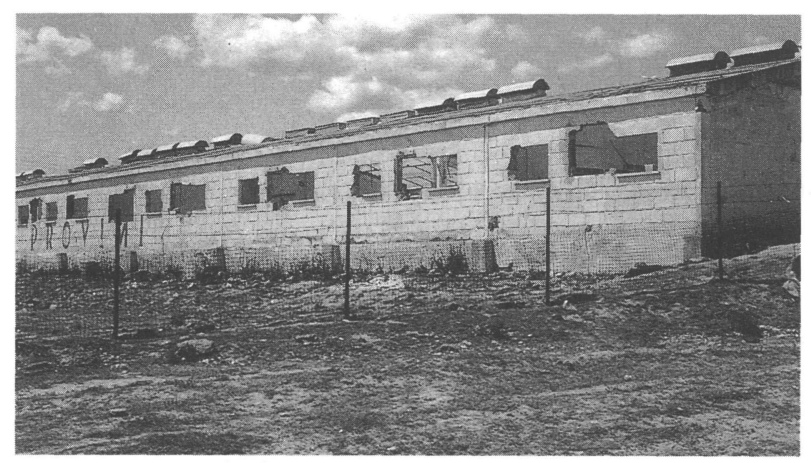

Uno de los problemas que se presentan con las instalaciones agrarias es el período siguiente a su vida útil. Hay terminos municipales donde las ruinas de las construcciones están cercanas a las cien. Por ello es necesario que la normativa tenga en cuenta los materiales y su posible degradación. En este aspecto deberían favorecerse las construcciones con tierra.

Montejo de la Sierra, Navalcarnero, Puebla de la Sierra, Redueña, Rozas de Puerto Real, San Martín de Valdeiglesias, Torrelaguna, Torrejón de Velasco, Valdemoro, Valdetorres del Jarama, Villanueva de la Cañada.

$2^{\circ}$ Grupo de aquellos que no tienen editadas (con anterioridad a octubre de 1992) las actuales Normas o el Plan General y por lo tanto las únicas que poseen editadas son las "Normas Subsidiarias y Complementarias" o "Proyecto de delimitación de suelo urbano y Normas sobre uso del suelo y edificación" datadas entre los años 1975-1979. A este grupo le llamaremos de aquí en adelante de Normas Subsidiarias antiguas frente al primero, que le llamaremos de Normas Subsidiarias actuales. En este segundo grupo están los siguientes municipios: Belmonte del Tajo, Campo Real, Casarrubuelos, Cervera de Buitrago, El Berrueco, El Atazar, Fresno del Torote, Gascones, La Acebeda, La Hiruela, La Serna del Monte, Manzanares el Real, Robregordo, Santa María de la Alameda, Somosierra, Titulcia, Valdepiélagos, Valdaracete, Villalbilla, Villavieja de Lozoya. En este segundo grupo las Normas que se han consultado son las normas que tienen editadas y son a las que haremos referencia, ya que algunos de ellos tienen Normas Subsidiarias que aun estando aprobadas no están editadas.

\section{ASPECTOS GENERALES DE LAS NORMAS}

Cuando se comparan las Normas Subsidiarias de los diversos municipios uno de los primeros datos que se observa es que de los núcleos que hemos llama-

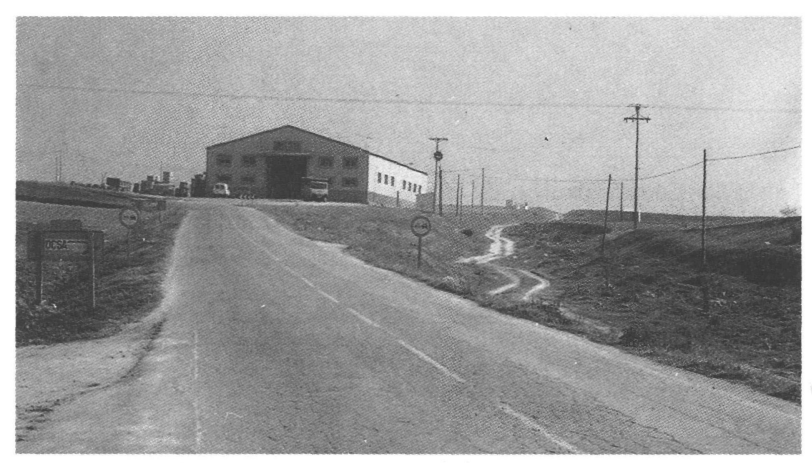

La ubicación de instalaciones en zonas de alta visibilidad, como son zonas elevadas o las entradas o salidas de los pueblos, deberian tener un tratamiento especial dentro de la normativa.

do rurales puros, que se seleccionaron (8 en total), sólo uno: Puebla de la Sierra tiene Normas actuales. Si se compara esto con las villas, se observa que de las 10 que estudiamos todas tienen actualizadas las Normas, faltando sólo Manzanares el Real ${ }^{(4)}$.

Esto nos lleva a pensar que aunque los objetivos de las Normas Subsidiarias son la defensa del patrimonio paisajístico y arquitectónico, prima realmente sólo lo segundo, ya que desde el punto de vista paisajístico, pocos son los que tendrían más interés que Manzanares el Real o que los núcleos rurales puros. Esto se ve confirmado por otra serie de hechos como son:

1 Existe al final de todas las Normas actuales un catálogo de elementos a proteger que sólo contienen elementos arquitectónicos y no parajes o elementos paisajísticos.

$2^{\circ}$ Si se compara la normativa de lo que hemos llamado condicionantes estéticos en suelo urbano, frente a la de suelo no urbanizable o con el no urbanizable especialmente protegido, hay una enorme diferencia a favor del suelo urbano, sobre todo por lo que se refiere al casco antiguo, como se puede observar en la tabla 1.

En el conjunto de las Normas también se observa que conforme son más actuales va aumentando tanto el estudio del paisaje, como el de los condicionantes estéticos.

Las N.S.P.M. (Normas Subsidiarias de PTaneamiento Municipal) se centran más en las normās de uso ${ }^{(5)}$ y en las condiciones de volumen que en el resto, 
Tabla 1.- Comparación de algunos condicionantes estéticos en 20 municipios de la Comunidad Autónoma de Madrid. En suelo urbano frente al suelo no urbanizable.

- Las normas generales afectan a suelo urbano y no urbanizable.

- Quedan reflejados los condicionantes si aparecen en algún sector o zona de suelo urbano frente a alguna zona del no urbanizable.

- Si: Si aparecen condicionantes.

- -: No aparecen condicionantes o de forma muy general.

\begin{tabular}{|c|c|c|c|c|c|c|c|c|c|c|c|c|c|c|c|c|c|c|c|c|c|c|}
\hline \multirow[b]{2}{*}{ Muncisero } & \multirow[b]{2}{*}{ NOPMAS } & \multirow[b]{2}{*}{$\begin{array}{l}\text { EDiF: } \\
\text { ALtu }\end{array}$} & \multicolumn{4}{|c|}{ CUBIERTA } & \multicolumn{5}{|c|}{ FACHADA } & \multirow{2}{*}{$\begin{array}{l}\text { (1) } \\
\text { TEX. } \\
\text { ELE. } \\
\text { EXT. }\end{array}$} & \multicolumn{3}{|c|}{ CERRAMIENTOS } & \multicolumn{4}{|c|}{ CARPATtEAA } & \multirow{2}{*}{$\begin{array}{c}\text { (3) } \\
\text { MPoos. } \\
0 \\
\text { MoLo. }\end{array}$} & \multirow{2}{*}{ persianas } & \multirow{2}{*}{$\begin{array}{l}\text { TOTAL } \\
\text { CONOAC }\end{array}$} \\
\hline & & & mat. & col. & inc. & $\begin{array}{l}\operatorname{cim}_{\text {ALE. }} \\
\text { AL. }\end{array}$ & MAT. & col. & 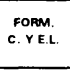 & $\underset{\substack{\operatorname{Dim}_{\text {HUE. }} \\
\text { UUE.V }}}{ }$ & DM. & & мат. & col. & Atr. & мar. & col. & TEX. & $\begin{array}{l}121 \\
\text { Fon } \\
\text { Dim. }\end{array}$ & & & \\
\hline $\begin{array}{l}\text { BATres } \\
1987\end{array}$ & \begin{tabular}{|l} 
N.o. \\
N.u. \\
N.N.u. \\
\end{tabular} & si & si & si & s! & s! & $\begin{array}{c}\mathrm{si} \\
\mathrm{si}\end{array}$ & $\begin{array}{l}\text { sl } \\
\text { si }\end{array}$ & $\mathrm{s!}$ & si & si & si & si & si & $\begin{array}{l}\text { si } \\
\text { si }\end{array}$ & si & si & si & sı & si & si & $\begin{array}{c}8 \\
18 \\
1 \\
\end{array}$ \\
\hline $\begin{array}{l}\text { BREA OEL TANO } \\
\text { 1987 T }\end{array}$ & \begin{tabular}{|l} 
No. \\
Nou \\
NNu
\end{tabular} & si & si & sı & si & si & si & si & s! & & & & & $\vdots$ & $\therefore$ & s! & si & & $\vdots$ & $\vdots$ & & : \\
\hline 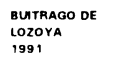 & $\begin{array}{l}\text { No. } \\
\text { N.u. } \\
\text { N.N. }\end{array}$ & si & $\begin{array}{l}\text { si } \\
\text { si }\end{array}$ & si & si & si & $\begin{array}{c}\mathrm{si} \\
\mathrm{sl}\end{array}$ & $\begin{array}{c}s \\
s i\end{array}$ & s! & si & $\begin{array}{l}\mathrm{sl} \\
\mathrm{sl}\end{array}$ & $\begin{array}{c}s ! \\
\text { si. }\end{array}$ & 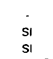 & st & $\begin{array}{l}\mathrm{si} \\
\mathrm{si}\end{array}$ & 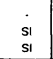 & 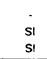 & s! & si & si & si & $\begin{array}{c}1 \\
20 \\
8\end{array}$ \\
\hline $\begin{array}{l}\text { cismpozunes } \\
19877\end{array}$ & $\begin{array}{l}\text { N.G. } \\
\text { N.u. } \\
\text { N.N. } \\
\end{array}$ & $\begin{array}{l}\mathrm{st} \\
\mathrm{si}\end{array}$ & s! & s! & s! & s! & s! & $s$ & s! & s! & st & $\begin{array}{l}\text { si. } \\
\text { si. }\end{array}$ & : & $\vdots$ & $\begin{array}{l}\mathrm{si} \\
\mathrm{si} \\
\end{array}$ & s! & s! & s! & s! & st & sı & $\begin{array}{l}17 \\
1 \\
3 \\
\end{array}$ \\
\hline $\begin{array}{l}\text { COBEÑA } \\
\text { } 1987\end{array}$ & $\begin{array}{l}\text { N.G. } \\
\text { N.u. } \\
\text { N.N.U. }\end{array}$ & $\begin{array}{c}\mathrm{si} \\
\mathrm{si}\end{array}$ & si & si & si & st & si & si & $\begin{array}{l}\text { si } \\
\text { s! }\end{array}$ & $\vdots$ & : & $\begin{array}{c}\text { si } \\
\text { si }\end{array}$ & & $\vdots$ & $\vdots$ & si & si & & $\vdots$ & $\therefore$ & : & $\begin{array}{r}2 \\
10 \\
2 \\
\end{array}$ \\
\hline $\begin{array}{l}\text { COMMEMAR DE } \\
\text { orta } \\
\text { 1985 }\end{array}$ & $\begin{array}{l}\text { No. } \\
\text { N.u. } \\
\text { N.N. } \\
\end{array}$ & $\begin{array}{l}\mathrm{sl} \\
\mathrm{si}\end{array}$ & si & $\therefore$ & $\therefore$ & sı & si & : & $\begin{array}{l}\mathrm{si} \\
\mathrm{si}\end{array}$ & si & si & & $\vdots$ & $\vdots$ & $\vdots$ & si & si & & si & $\vdots$ & $\vdots$ & $\begin{array}{l}3 \\
8 \\
1 \\
\end{array}$ \\
\hline $\begin{array}{l}\text { CHANCHON } \\
1985 \\
\end{array}$ & $\begin{array}{l}\text { N.o. } \\
\text { N.u. } \\
\text { N.N. } \\
\end{array}$ & $\begin{array}{l}\mathrm{si} \\
\mathrm{si}\end{array}$ & $\begin{array}{l}\mathrm{si} \\
\mathrm{si} \\
\mathrm{si}\end{array}$ & $\begin{array}{l}\mathrm{si} \\
\mathrm{si} \\
\end{array}$ & s! & si & $\begin{array}{l}\mathrm{sl} \\
\mathrm{si}\end{array}$ & $\begin{array}{c}\mathrm{si} \\
\mathrm{si}\end{array}$ & s! & $\vdots$ & si & : & si & si & $\begin{array}{l}\mathrm{sl} \\
\mathrm{sl}\end{array}$ & si & si & & s! & : & $\dot{s i}$ & $\begin{array}{c}3 \\
14 \\
5\end{array}$ \\
\hline $\begin{array}{l}\text { ESTREMERA } \\
1988\end{array}$ & $\begin{array}{l}\text { N.G. } \\
\text { N.u. } \\
\text { N.N. } \\
\end{array}$ & $\begin{array}{c}\mathrm{si} \\
\mathrm{si}\end{array}$ & $\begin{array}{c}\mathrm{si} \\
\mathrm{si} \\
\end{array}$ & $\dot{s i}$ & $\begin{array}{c}\mathrm{si} \\
\mathrm{si}\end{array}$ & si & $\begin{array}{c}\mathrm{sl} \\
\mathrm{ss} \\
\end{array}$ & $\begin{array}{c}\mathrm{si} \\
\mathrm{si} \\
\mathrm{si}\end{array}$ & s! & si & si & : & : & : & si & si & si & & s! & si & si & $\begin{array}{c}2 \\
13 \\
6 \\
\end{array}$ \\
\hline $\begin{array}{l}\text { HORCANO DE LA } \\
\text { SIERAAA } \\
1986\end{array}$ & $\begin{array}{l}\text { N.G. } \\
\text { N.u. } \\
\text { N.N.U. }\end{array}$ & si & si & si & s! & $s !$ & sı & si & s! & : & $\therefore$ & & : & $:$ & . & & & & s! & : & & $\begin{array}{l}4 \\
5 \\
0\end{array}$ \\
\hline 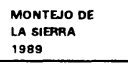 & \begin{tabular}{|l} 
N.G. \\
N.u. \\
N.N.u. \\
\end{tabular} & $\begin{array}{c}\mathrm{sl} \\
\mathrm{sl}\end{array}$ & $\begin{array}{l}\mathrm{sl} \\
\mathrm{ss} \\
\end{array}$ & $\begin{array}{l}\mathrm{sl} \\
\mathrm{s} \\
\mathrm{s}\end{array}$ & $\begin{array}{l}\mathrm{si} \\
\mathrm{si} \\
\mathrm{si}\end{array}$ & $\begin{array}{l}\mathrm{sl} \\
\mathrm{s!} \\
.\end{array}$ & $\begin{array}{l}\mathrm{sl} \\
\mathrm{ss}\end{array}$ & $\begin{array}{l}\mathrm{st} \\
\mathrm{si}\end{array}$ & $\begin{array}{l}\mathrm{sl} \\
\mathrm{si}\end{array}$ & s! & $\therefore$ & & si & si & $\begin{array}{l}\mathrm{sl} \\
\dot{s} \\
\end{array}$ & $\begin{array}{l}\mathrm{si} \\
\mathrm{si}\end{array}$ & $\begin{array}{l}\mathrm{si} \\
\mathrm{si}\end{array}$ & : & s! & si & & $\begin{array}{l}5 \\
11 \\
11\end{array}$ \\
\hline $\begin{array}{l}\text { NavaLCARNERO } \\
1981\end{array}$ & \begin{tabular}{|l} 
No. \\
N.u. \\
N.N.U.
\end{tabular} & $\begin{array}{l}\mathrm{sl} \\
\mathrm{sl}\end{array}$ & & & $\begin{array}{l}\mathrm{si} \\
\mathrm{si}\end{array}$ & : & si & $\dot{\text { si }}$ & s! & $\therefore$ & si & : & si & $\vdots$ & si & & : & : & . & $\therefore$ & & 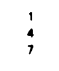 \\
\hline 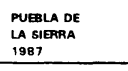 & \begin{tabular}{|l} 
No. \\
N.u. \\
N.N.u. \\
\end{tabular} & $\begin{array}{l}\mathrm{si} \\
\mathrm{si} \\
\end{array}$ & si & $\vdots$ & $\begin{array}{c}s i \\
s i \\
s i\end{array}$ & $\begin{array}{l}\mathrm{sl} \\
\mathrm{s!}\end{array}$ & si & si & s! & : & : & $\dot{s i}$ & $\vdots$ & $\vdots$ & $\begin{array}{l}\text { s! } \\
s i \\
\end{array}$ & si & s! & s! & . & st & & $\begin{array}{l}4 \\
8 \\
4\end{array}$ \\
\hline $\begin{array}{l}\text { REDUEÑ } \\
\text { 1988 }\end{array}$ & $\begin{array}{l}\text { No. } \\
\text { Nou. } \\
\text { N.N.u. }\end{array}$ & $\begin{array}{c}\mathrm{sl} \\
\mathrm{si}\end{array}$ & $\begin{array}{c}\text { si } \\
\text { si }\end{array}$ & $\begin{array}{l}\mathrm{sl} \\
\mathrm{si}\end{array}$ & $\vdots$ & $\mathrm{s!}$ & si & : & s! & : & : & $\vdots$ & $\vdots$ & $\vdots$ & s! & $\vdots$ & . & : & $\mathrm{sl}$ & $\vdots$ & : & 4 \\
\hline $\begin{array}{l}\text { ROZAAS DE } \\
\text { PUPBOT REAL } \\
1984\end{array}$ & $\begin{array}{l}\text { N.o. } \\
\text { N.u. } \\
\text { N.N. }\end{array}$ & $\begin{array}{l}\mathrm{sl} \\
\mathrm{sl} \\
\mathrm{sl}\end{array}$ & s! & st & : & $\dot{s i}$ & & & si & : & $\vdots$ & : & : & : & : & : & : & : & $\therefore$ & : & : & $\begin{array}{l}3 \\
3 \\
1 \\
\end{array}$ \\
\hline 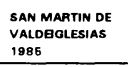 & $\begin{array}{l}\text { No. } \\
\text { N.u. } \\
\text { N.N.U. }\end{array}$ & $\begin{array}{c}\text { si } \\
\text { si }\end{array}$ & $\vdots$ & si & $\vdots$ & s! & $\vdots$ & . & s! & $\vdots$ & $\vdots$ & $\vdots$ & si & : & s! & & $\vdots$ & & s! & $\vdots$ & . & $\begin{array}{l}4 \\
2 \\
5 \\
\end{array}$ \\
\hline $\begin{array}{l}\text { PORяваaUNa } \\
\text { 1987 }\end{array}$ & $\begin{array}{l}\text { N.o. } \\
\text { N.u. } \\
\text { N.N.U. }\end{array}$ & $\begin{array}{c}\text { si } \\
\text { si } \\
\text { sins }\end{array}$ & si & & s! & si & $\begin{array}{c}s ! \\
s i\end{array}$ & $\begin{array}{l}\mathrm{sl} \\
\mathrm{si}\end{array}$ & si & $\vdots$ & $\begin{array}{l}\mathrm{si} \\
\mathrm{si}\end{array}$ & si: & $\begin{array}{c}s 1 \\
\text { si }\end{array}$ & $\begin{array}{l}s ! \\
s i\end{array}$ & $\vdots$ & $\begin{array}{l}\mathrm{sl} \\
\mathrm{si}\end{array}$ & $\begin{array}{l}\mathrm{sl} \\
\mathrm{si}\end{array}$ & : & s! & : & $\therefore$ & $\begin{array}{c}8 \\
12 \\
2 \\
\end{array}$ \\
\hline $\begin{array}{l}\text { OOREEON DE } \\
\text { VEAASCO } \\
\text { c990 }\end{array}$ & $\begin{array}{c}\text { No. } \\
\text { N.u. } \\
\text { N.N.u. }\end{array}$ & $\begin{array}{l}\mathrm{sl} \\
\mathrm{si} \\
\mathrm{si}\end{array}$ & $\begin{array}{c}\mathrm{sl} \\
\mathrm{sl}\end{array}$ & $\begin{array}{l}\mathrm{sl} \\
\mathrm{sl}\end{array}$ & $\begin{array}{l}\mathrm{sl} \\
\mathrm{sl} \\
\mathrm{ss} \\
\mathrm{s}\end{array}$ & $\begin{array}{l}\mathrm{si} \\
\mathrm{si}\end{array}$ & $\dot{s i}$ & si & $\begin{array}{l}\text { si } \\
\text { si }\end{array}$ & si & $\begin{array}{l}\mathrm{sl} \\
\mathrm{sl}\end{array}$ & si & $\begin{array}{l}\text { s! } \\
\text { s! }\end{array}$ & $\begin{array}{l}\mathrm{sl} \\
\mathrm{sl}\end{array}$ & $\begin{array}{l}\mathrm{s} \\
\mathrm{s} s \\
\mathrm{~s} \\
\mathrm{~s}\end{array}$ & s! & s! & si & s! & si & si & $\begin{array}{l}10 \\
18 \\
4 \\
\end{array}$ \\
\hline $\begin{array}{l}\text { Valogmoro } \\
\text { 1987 }\end{array}$ & \begin{tabular}{|l} 
No. \\
N. \\
N.N.U. \\
\end{tabular} & $\begin{array}{c}\mathrm{sl} \\
\mathrm{s} \\
\mathrm{s} \\
\mathrm{si}\end{array}$ & $\vdots$ & & $\begin{array}{c}\text { si } \\
\text { st }\end{array}$ & s! & $\vdots$ & . & $\begin{array}{l}51 \\
\mathrm{~s} \\
\mathrm{~s}\end{array}$ & : & & si. & $\vdots$ & $\vdots$ & $\begin{array}{l}\mathrm{sl} \\
\mathrm{si} \\
\mathrm{sl}\end{array}$ & $\vdots$ & $\vdots$ & $\vdots$ & si & $\vdots$ & $\vdots$ & $\begin{array}{l}4 \\
4 \\
\end{array}$ \\
\hline $\begin{array}{l}\text { ValoETORRES } \\
\text { DEL JARARA } \\
\text { 1987 }\end{array}$ & $\begin{array}{l}\text { No. } \\
\text { N.u. } \\
\text { N.N.U. }\end{array}$ & 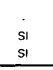 & $\begin{array}{l}\mathrm{si} \\
\mathrm{si} \\
\mathrm{si}\end{array}$ & $\begin{array}{l}s 1 \\
\text { si } \\
s\end{array}$ & $\begin{array}{c}\text { si } \\
\text { si }\end{array}$ & $s !$ & $\begin{array}{l}\mathrm{sl} \\
\mathrm{si} \\
\mathrm{s}\end{array}$ & $\begin{array}{l}\mathrm{sl} \\
\mathrm{st}\end{array}$ & s! & & & & si & $\vdots$ & $\begin{array}{l}\text { s! } \\
\text { si }\end{array}$ & si & si & & s! & : & : & $\begin{array}{l}4 \\
8 \\
8\end{array}$ \\
\hline $\begin{array}{l}\text { VILAAUEEVA DE } \\
\text { LA CAANADA } \\
\text { 1987 }\end{array}$ & $\begin{array}{l}\text { N.G. } \\
\text { N.u. } \\
\text { N.N.U. }\end{array}$ & $\begin{array}{c}\mathrm{si} \\
\mathrm{si}\end{array}$ & $\begin{array}{c}\mathrm{si} \\
\mathrm{si}\end{array}$ & $\vdots$ & $\vdots$ & s! & $\begin{array}{c}\mathrm{si} \\
\mathrm{si} \\
\mathrm{si}\end{array}$ & si & $\mathrm{s!}$ & & & & si & $\vdots$ & $\begin{array}{l}\mathrm{sl} \\
\mathrm{si} \\
\mathrm{si}\end{array}$ & sı & si & & s! & : & & $\begin{array}{l}4 \\
6 \\
9\end{array}$ \\
\hline
\end{tabular}

\section{SÍMBOLOS}

N. G. = Normas generales.
N. U. = Normas particulares suelo urbano.
N. N. U. = Normas particulares suelo no urbanizable.
EDIF. ALTU. = Altura de la edificación.
MAT. = Material.
COL. = Color.
INC. = Inclinación.
DIM. ALE. = Dimensión aleros.

FORM. C. y EL. = Forma de cuerpos y elementos salientes.

DIM. HUE. V. = Dimensión huecos ventana.

DIM. HUE. $\mathbf{P}$. = Dimensión huecos puertas.

TEX. ELE. EXT. = Textura elementos exteriores

TEX. $=$ Textura .

FOR. DIM. $=$ Forma. Dimensión

IMPOS. O MOLD. = Impostas o molduras.

TOTAL CONDIC $=$ Total condiciones

(1) Si se especifica textura en algun elemento exterior. Si senala "es que se probibe texturas brillantes en todo el elemento exterior. (2) Si se especifican forma o dimensión de algunos elementes de caryinteria. (3) Si see especifican forma o dimensión de impostas o de molduras. (4) Si se especifican forma. dimensión o colores de las persianas. 
incluso las antiguas tratan extensamente estos dos aspectos. En los municipios con Normas actuales, se observa un gran desarrollo de las normas de uso; así el suelo urbano se suele clasificar en diversos sectores (casco antiguo, equipamientos, zonas verdes, ensanche, etc.); dentro de cada uno de ellos se definen diversos tipos de uso, normalmente: principal o característico ${ }^{(6)}$; compatible, admisible, tolerado o complementario ${ }^{(7)}$ y prohibido $^{(8)}$ (ver los municipios de: Batres, Brea del Tajo, Buitrago de Lozoya, Cobeña, Montejo de la Sierra, Navalcarnero, Puebla de la Sierra, Redueña, Torrelaguna, Torrejón de Velasco, Valdetorres del Jarama y Villanueva de la Cañada).

En otros casos se llegan a definir 5 tipos de usos: predominante, compatible, complementario, condicionado y prohibido (N.S.P.M. de Chinchón, y Estremera). Lo que da lugar a que en cada zona se tenga que asignar cada uno de los diversos tipos de usos, ocasionando en algunos casos decenas de posibilidades ${ }^{(1)}$.

La profusión con que se desarrollan las normas de uso y de volumen parece indicar que se están imitando planes de urbanismo de grandes ciudades, donde la presión inmobiliaria es grande, situación que no tiene sentido en un municipio de unos centenares de vecinos.

\section{CONDICIONANTES ESTÉTICOS DE LAS NORMAS}

Entendemos por condicionantes estéticos aquellos que se refieren a los atributos que hemos llamado artísticos, es decir los que se refieren a: la forma, color, la textura y los materiales.

Las Normas suelen distinguir entre condicionantes de volumen y los condicionantes "estéticos". En la clasificación propuesta en este trabajo, la altura y la pendiente de la cubierta lo consideraremos como condicionante estético, aunque en las Normas suelen incluirse dentro del grupo de condicionantes de volumen. Estos, los condicionantes de volumen, ya desde las primeras Normas (año 1975) aparecen bastante desarrollados, fijándose la altura máxima, la parcela mínima edificable, la densidad máxima sobre la parcela, etc.

El resto de los condicionantes estéticos, apenas están concretados en las Normas antiguas, siendo normalmente comunes a todos los municipios de este segundo grupo. Se especifican en cuatro apartados: uno de forma general y los otros tres aplicables a: suelo rústico; suelo de reserva metropolitana o no urbanizable común (dependiendo si son Normas Complementarias o Delimitación de casco urbano); y casco antiguo.

En concreto se establece en las Normas antiguas:

$1^{\circ}$ De forma general:

"Toda construcción, cualquiera que sea su destino o situación deberá satisfacer las condiciones establecidas con carácter general en el Art. 73 y concordantes de la Ley del Suelo. Las afectadas por declaraciones oficiales de monumentos o conjuntos se sujetarán a sus regulaciones especificas".

2o Condiciones estéticas para suelo rústico:

"Las edificaciones se construirán en todo su perimetro con los materiales apropiados a su carácter y destino y los cerramientos de las parcelas serán preferentemente de piedra, setos verdes o al menos de elementos resistentes que no se deterioren por agentes atmosféricos".

$3^{\text {o }}$ Condiciones relativas al Suelo de reserva metropolitana o Suelo no urbanizable común:

"Las edificaciones se construirán en todo su perimetro con los materiales apropiados en su carácter y destino de forma que no desentonen en su emplazamiento y los cerramientos de las parcelas serán preferentemente de piedra, setos verdes o, al menos, de elementos resistentes que no se deterioren por agentes atmosféricos".

$4^{\circ}$ Condiciones del casco antiguo:

"Se observarán por cada tramo de calle o espacio público y referidas a la edificación con más de 50 años de antigüedad, las siguientes condiciones:

- Los módulos de edificación se manifestarán por volumen y ancho y composición de fachada, siguiendo los ritmos existentes.

- Con carácter específico para el casco antiguo, la altura de cornisa y la profundidad de la edificación se obtendrá como media aritmética del total de las unidades de cada calle. 
- Se permitirán vuelos, si los bubiese en la edificación antigua, con soluciones semejantes a las existentes en ellos, probibiéndose en todo caso los cuerpos volados cerrados.

- En las fachadas y en las cubiertas se emplearán formas, texturas y colores con gamas acordes con las soluciones existentes en los ambientes respectivos.

- En caso de reformas urbanas, se respetará el arbolado y los elementos arquitectónicos existentes en calles y plazas, integrándolos en las nuevas soluciones".

En las Normas actuales el grado de desarrollo de las condiciones estéticas en el suelo urbano y en concreto en el casco antiguo ha sido muy grande, así por ejemplo en Buitrago de Lozoya (1991) el apartado de "condiciones estéticas" para el casco histórico ocupa una decena de páginas, sólo las condiciones de la fachada son 4 páginas.

Entre la normativa propuesta para Buitrago y las de los años 70 hay una amplia gama de municipios todos incluidos en lo que hemos llamado primer grupo de Normas o Normas actuales, en éstas, aunque el desarrollo de los condicionantes estéticos no sea tan extenso, sí suelen especificarse con cierto detalle.

A pesar del avance realizado en el estudio de los condicionantes estéticos, éstos sólo se suelen determinar para el suelo urbano y es raro que se definan con la misma precisión para el suelo no urbanizable. Esto pensamos que es debido, como ya hemos expuesto, a una mayor preocupación por temas arquitectónicos o urbanísticos ${ }^{(10)}$ que por los paisajísticos.

\section{COMPARACIÓN DE LOS CONDICIONANTES ESTÉ- TICOS EN SUELO URBANO Y NO URBANIZABLE}

Con el fin de poder comparar el desarrollo de los condicionantes estéticos en suelo urbano frente al suelo no urbanizable, en las Normas actuales se ha elaborado la tabla 1.

Para la elaboración de la tabla 1 se han elegido 20 variables, entre las que consideramos que definen aspectos estéticos. Se han elegido por ser las que con más frecuencia suelen aparecer y que son un

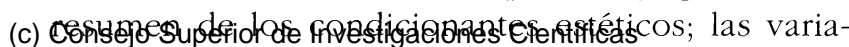
Licencia Creative Commons 3.0 España (by-nc) bles elegidas son:

- Altura del edificio.

- Cubierta:

+ Materiales.

+ Color.

+ Forma y dimensiones; se han seleccionado como características más importantes: la inclinación de la cubierta y la longitud del alero.

- Fachada:

+ Materiales.

+ Color.

+ Forma y dimensiones; las variables elegidas como más representativas han sido: la forma y dimensiones de los elementos y cuerpos salientes; las dimensiones y forma de los huecos de entrada, y las de los huecos para ventanas.

- Cerramientos:

+ Materiales.

+ Color.

+ Forma y dimensiones caracterizadas por la altura del cerramiento.

- Carpintería:

+ Materiales.

+ Color.

+ Textura

+ Forma y dimensiones: se señala si se especifica forma o dimensiones de algunos elementos de la carpintería.

- Detalles:

Se ha elegido la caracterización de persianas y de impostas o molduras.

- Textura:

Hemos puesto aparte la textura ya que sólo se suele especificar para el conjunto de elementos y en concreto prohibiendo las texturas brillantes u obligando a la utilización de texturas mates para todos los elementos exteriores. No obstante en la carpintería se da con alguna frecuencia la prohibición de los colores brillantes, por ello se ha separado la textura de la carpintería de la textura en conjunto; la primera parece hacer más bien referencia a los detalles mientras que la segunda hace referencia al conjunto de materiales. http://informesdelaconstruccion.revistas.csic.es 
En la tabla 1 están reflejadas las 20 variables elegidas; como ya se ha dicho se comparan los condicionantes estéticos en suelo urbano frente al suelo no urbanizable, por ello aparece un signo - cuando no vienen especificados y se señala con SI cuando vienen fijados. Se considera que vienen determinados si aparece en alguna ordenanza o zona del correspondiente suelo (urbano o no urbanizable). No hemos considerado que está especificado si aparece de forma muy general, así por ejemplo "se utilizarán colores, texturas y materiales acordes con el entorno".

En bastantes casos las Normas determinan ciertos condicionantes para todo el municipio sin fijar la zona donde se aplica, así por ejemplo muchas de las especificaciones que aparecen en las normas generales de la edificación, en este caso los hemos agrupado en la fila de normas generales. Así pues para cada municipio tenemos tres filas: una para normas generales, otra para normas particulares para suelo urbano y la tercera para normas particulares para suelo no urbanizable.

Con los datos que se establecen en la tabla 1 podemos calcular la media de condicionantes sobre los 20 máximos que aparecen por cada tipo de suelo dándonos los siguientes resultados:

- Media para suelo urbano: 8,9.

- Media para suelo no urbanizable: 4,6.

- Media para normas generales (comunes para suelo urbano y no urbanizable): 4,4 .

Como se observa hay una enorme desproporción entre los condicionantes "urbanos" y "no urbanizables", estos últimos aparecen al nivel de las normas generales. En la tabla 1 podemos calcular además cuántos municipios fijan cada una de las variables y de esta forma hacer una clasificación por orden de importancia de los diversos parámetros, así tendríamos:

1을 Altura del edificio (17 municipios).

2o Dimensiones de cuerpos y elementos salientes (16 municipios).

3 o Inclinación de las cubiertas (15 municipios).

$4^{\circ}$ Altura de los cerramientos (13 municipios).

$4^{\circ}$ Dimensiones de los aleros (13 municipios).

$4^{\circ}$ Forma y dimensiones de la carpintería (13 municipios).

$5^{\circ}$ Materiales de la fachada (12 municipios).

6o Materiales de la cubierta (10 municipios).

$7^{\circ}$ Color de la cubierta ( 9 municipios).

7 o Color de la fachada (9 municipios). $7^{\circ}$ Materiales del cerramiento (9 municipios).

$8^{\circ}$ Textura de elementos exteriores (7 municipios).

$9^{\circ}$ Color del cerramiento ( 5 municipios).

$10^{\circ}$ Dimensiones de huecos de puerta ( 4 municipios).

$10^{\circ}$ Materiales de carpintería ( 4 municipios).

$10^{\circ}$ Color de la carpintería ( 4 municipios).

$11^{\circ}$ Dimensiones hueco ventanas (2 municipios).

$12^{\circ}$ Textura carpintería (1 municipio).

$12^{\circ}$ Impostas o molduras (1 municipio).

$12^{\circ}$ Persianas (1 municipio).

Sobre la relación anterior se pueden hacer las siguientes observaciones:

- Con relación a las dimensiones de cuerpos y elementos salientes: estas condiciones se fijan en las normas generales y por tanto son aplicables al suelo no urbanizable, pero parecen estar más en relación con el núcleo urbano ya que se suelen fijar en función del ancho de la calle. Normalmente se permiten unos salientes máximos de entre 0,40 y $0,60 \mathrm{~m}$. El mismo planteamiento suele hacerse con relación a la dimensión de los aleros, en donde el vuelo máximo suele estar entre 0,30 y $0,40 \mathrm{~m}$.

- Por lo que se refiere a forma y dimensiones de la carpintería ésta suele aludir a dimensiones de las rejas de las ventanas, o en general a las protecciones de los huecos de los edificios, con lo que se especifica más por razón de protección que estética; de hecho en la mayor parte de los municipios aparecen dentro del capítulo de protecciones y no de normas estéticas (Horcajo de la Sierra, Montejo de la Sierra, Puebla de la Sierra, San Martín de Valdeiglesias y Villanueva de la Cañada).

- Con relación a los materiales y colores del cerramiento, la mayor parte vienen fijados al determinarse los materiales y colores de la fachada, ya que se suele especificar como materiales y colores de los paramentos verticales (Montejo de la Sierra, Navalcarnero, Redueña, Valdetorres del Jarama, Villanueva de la Cañada), por ello lo estudiaremos en general como características de la fachada.

Por estas observaciones pensamos que los elementos estéticos más importantes en las Normas son los siguientes siete: (numerados por orden de importancia).

1ํAtura del edificio.

$2^{\circ}$ Inclinación de la cubierta. 
$3^{\text {o }}$ Altura de los cerramientos.

4ํㅡㄹ Materiales de la fachada.

5 Materiales de la cubierta.

$6^{\circ}$ Color de la fachada.

6o Color de la cubierta.

Veamos los elementos más característicos de cada uno de estos siete:

* Materiales: en la cubierta (ver tablas: 2 y 3) y la fachada (ver tablas: 4 y 5). En cuanto a la cubierta se recomienda la utilización de teja (10 municipios). En 9 municipios se prohíbe o se limita el uso del fibrocemento.

Por lo que se refiere a los materiales de la fachada se suele prohibir el ladrillo (tabla 4) y el bloque de hormigón en algunos municipios (tabla 5).

* Textura: se especifica la prohibición de utilizar materiales brillantes o reflactantes para cualquier elemento o revestimiento exterior (ver tabla 6). Son 7 los municipios que lo prohiben.

* Forma y dimensiones: altura del cerramiento (tabla 8); altura de las edificaciones (tabla 8); inclinación de la cubierta (ver tabla 9). Por lo que se refiere a la altura del cerramiento oscila entre $1 \mathrm{~m}$ y $3 \mathrm{~m}$, aunque lo normal es 1,5 m también se suele especificar la altura de la parte opaca. En total son 13 los municipios que especifican la altura del cerramiento.

En cuanto a la altura de las edificaciones ésta suele variar (ver tabla 7 ) entre $2,5 \mathrm{~m}$ y $10,5 \mathrm{~m}$, aunque algunas de estas variaciones se deban a la forma de medir la altura. En total son 17 municipios que especifican la altura de la edificación.

La inclinación de las cubiertas es el elemento más especificado en las Normas para el suelo no urbanizable después de la altura de la edificación, en total son 15 municipios los que fijan la inclinación de la cubierta (ver tabla 9). Por lo que se refiere a los municipios estudiados podemos agruparlos en tres:

1ㅂ Grupo; municipios en que está prohibido la utilización de cubiertas planas ( 5 municipios).
Tabla 2.- Exigencia de la utilización de la teja en los diversos municipios de Madrid según las normas subsidiarias en el suelo no urbanizable ${ }^{\bullet}$.

\begin{tabular}{||l|l||}
\hline \multicolumn{2}{|c||}{ EXIGEN } \\
\hline TEJA CURVA & Buitrago \\
\hline TEJA O SIMILAR & Rozas de Puerto Real \\
\hline TEJA COLOR NATURAL & $\begin{array}{l}\text { Brea del Tajo } \\
\text { Montejo de la Sierra } \\
\text { Navalcarnero } \\
\text { Redueña } \\
\text { Villanueva de la Cañada } \\
\text { Valdetorres del Jarama }\end{array}$ \\
\hline TEJA COLOR ROJO O SIMILAR & $\begin{array}{l}\text { Ciempozuelos } \\
\text { Torrejón de Velasco }\end{array}$ \\
\hline \multicolumn{2}{|c||}{ PROIIBBEN } \\
\hline $\begin{array}{l}\text { TEJAS O PLACA DE CUBIERTA DE COLOR DIFERENTE } \\
\text { AL NATURAL DE LA CERÁMICA }\end{array}$ & $\begin{array}{l}\text { Chinchón } \\
\text { San Martín de Valdeiglesias }\end{array}$ \\
\hline
\end{tabular}

- Se considera que especifican para suelo no urbanizable en material de cubierta u otros parámetros. si en alguna zona de este tipo de suelo se queda fijado el tipo de material (o los parámetros) o si viene especificada en alguna norma general que se apli-
que al suelo no urbanizable.

Tabla 3.- Municipios que en sus N.S.P.M ${ }^{\bullet}$ especifican en el suelo no urbanizable la utilización del fibrocemento como material de cubierta.

\begin{tabular}{|c|c|}
\hline \multicolumn{2}{|l|}{ PROIIIBEN } \\
\hline FIBROCEMENTO & Buitraggo \\
\hline FIBROCEMENTO TRANSLÚCIDO & San Martín de Valdeiglesias \\
\hline FIBROCEMENTO DE COLOR GRIS & $\begin{array}{l}\text { Chinchón } \\
\text { Estremera } \\
\text { San Martin de Valdeiglesias }\end{array}$ \\
\hline \multicolumn{2}{|l|}{ PERMITEN } \\
\hline $\begin{array}{l}\text { FIBROCEMENTO CON TRATAMIENTO DE COLORES TERROSOS } \\
\text { Y OCRES }\end{array}$ & $\begin{array}{l}\text { Montejo de la Sierra } \\
\text { Navalcarnero } \\
\text { Redueña } \\
\text { Valdetorres del Jarama }\end{array}$ \\
\hline $\begin{array}{l}\text { FIBROCEMENTO. Sólo en ejecuciones provisionales en las } \\
\text { edificaciones de poligonos industriales }\end{array}$ & Torrejón de Velasico \\
\hline
\end{tabular}

- N.S.P.M. Siglas de las Normas Subsidiarias de Planteamiento Municipal, algunas veces las referenciamos como Normas subsidiarias.

Tabla 4.- Municipios que en sus N.S.P.M. especifican en suelo no urbanizable la utilización del ladrillo en la fachada.

\begin{tabular}{||c|l||}
\hline \multicolumn{2}{|c|}{ PROIIBEN } \\
\hline LADRILLO VISTO & $\begin{array}{l}\text { Buitrago } \\
\text { Chinchón } \\
\text { Estremera }\end{array}$ \\
\hline LADRILLO CON ACABADO PROPIO DE CARA OCULTA & Valdemoro \\
\hline LADRILLOS BLANCOS, VETEADOS O VIDRIADOS & Ciempozuelos \\
\hline \multicolumn{2}{|c||}{ PERMITE } \\
\hline \begin{tabular}{l|l||} 
LADRILLO VISTO CERÁMICO. ROJIZO Y OCRE EN SU \\
COLOR NATURAL
\end{tabular} & Ciempozuelos \\
\hline \multicolumn{2}{|c||}{ EXIGEN } \\
\hline LADRILLO PARDO DE USO LOCAL \\
\hline
\end{tabular}

- Sólo se permiten fachadas vistas cuando se realicen en fábrica de ladrillo pardo de uso local.

Tabla 5.- Municipios que en sus N.S.P.M. prohiben en suelo no urbanizable el bloque de hormigón en la fachada.

\begin{tabular}{|l|l|}
\hline BLOQUE DE HORMIGÓN VISTO DE COLOR GRIS & Chinchón \\
\hline $\begin{array}{l}\text { BLOQUE DE HORMIGÓN CON ACABADO PROPIO DE CARA } \\
\text { OCULTA }\end{array}$ & Valdemoro \\
\hline
\end{tabular}

Tabla 6.- Municipios que en sus N.S.P.M. prohiben la textura brillante para cualquier elemento o revestimiento exterior en suelo no urbanizable.

\begin{tabular}{|l|l|}
\hline $\begin{array}{l}\text { Buitrago de Lozoya } \\
\text { Ciempozuelos } \\
\text { Cobeñid } \\
\text { Puella de la Sierra }\end{array}$ & $\begin{array}{l}\text { Torrelaguna } \\
\text { Torrejón de Velasco } \\
\text { Valdemoro }\end{array}$ \\
\hline
\end{tabular}


Tabla 7.- Municipios que en sus N.S.P.M., en suelo no urbanizable, especifican la altura del cerramiento:

\begin{tabular}{|c|c|}
\hline 1 m Máximo: & $\begin{array}{l}\text { Montejo de la Sierra (S.P.E.P.) } \\
\text { Villanueva de la Cañada (S.P.E.P.) }\end{array}$ \\
\hline 1 m Máximo para la parte opaca: & $\begin{array}{l}\text { Batres } \\
\text { Buitrago de Lozoya } \\
\text { Ciempozuelos } \\
\text { Cobeña } \\
\text { Puebla de la Sierra } \\
\text { Torrejón de Velasco } \\
\end{array}$ \\
\hline 1,20 m Máximo: & $\begin{array}{l}\text { Ciempozuelos (S.P.E.E.) } \\
\text { en (S.P.E.P.) } \\
\text { Puebla de la Sierra y } \\
\text { Torrejón de Velasco }\end{array}$ \\
\hline 1,50 m Máximo: & $\begin{array}{l}\text { Montejo de la Sierra } \\
\text { Navalcarnero } \\
\text { Redueña } \\
\text { Valdetorres del Jarama } \\
\text { Villanueva de la Cañada }\end{array}$ \\
\hline $2 \mathrm{~m}$ Máximo y sólo $0,20 \mathrm{~m}$ opaco: & Torrejón de Velasco \\
\hline $2 \mathrm{~m}^{\bullet \bullet:}$ & $\begin{array}{l}\text { San Martín de Valdeiglesias } \\
\text { Chinchón }\end{array}$ \\
\hline 3 m Máximo: & Valdemoro \\
\hline
\end{tabular}

- Abreviaturas: (de las tablas 7 y 8 )

A.C.: Zona de acampada.

A.P.: $\quad$ Áreas de protección de masas arbóreas, áreas de protección de contami-

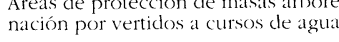

S.P.E.A.: Suelo no urbanizable de especial protección por su interés adropecuario.

S.P.E.D.: Suelo no urbanizable de protección especial dehesal.

S.P.E.E.: Suelo no urbanizable de especial protección por su interés ecológico.

S.P.E.F.: Suelo no urlanizable de protección especial forestal

S.P.E.P.: $\quad$ Suelo no urbanizable de especial protección por su interés paisajijistico. Entre paréntesis si se especifica para alguna zona del suelo no urbanizable

- Se determina para cerramientos de solares en estos dos municipios, que por no tener normativa específica para el suelo no urbanizahle, podría aplicarse en algunas instalaciones situadas en este tipo de suelo.

2o Grupo; municipios en que se fija un máximo de inclinación (10 municipios).

$3^{\text {er }}$ Grupo; municipios que determinan una inclinación máxima y mínima. (3 municipios).

* Colores: se suelen fijar para la cubierta o elementos de cobertura (ver tabla 10) y para la fachada o paramentos verticales (ver tabla 11).

Por lo que se refiere a la cubierta se suele recomendar el color rojo ( 3 municipios) o los colores ocres (5 municipios), prohibiéndose el fibrocemento de color gris ( 3 municipios).

El color en la fachada tiene más desarrollo que en la cubierta, aunque el número de municipios que lo estudian sea el mismo en una que en otra. El color blanco en algunos está prohibido (Buitrago) mientras que en otros está recomendado (Estremera y Ciempozuelos). El color gris y el negro están prohibidos (2 municipios). La práctica totalidad de los municipios parece estar de acuerdo en la utilización de los colores ocres (8 municipios sobre 9).
Tabla 8.- Municipios que en sus N.S.P.M., en suelo no urbanizable, especifican la altura máxima de construcción'.

\begin{tabular}{|c|c|}
\hline $2,5 \mathrm{~m}:$ & San Martin de Valdeiglesias (A.C.) (6*) \\
\hline $3 \mathrm{~m}:$ & $\begin{array}{l}1 \text { planca (6*) Montejo de la Sierra (S.P.E.P) } \\
\text { Villanueva de la Cañada (8*) (S.P.E.P) }\end{array}$ \\
\hline $3.5 \mathrm{~m}:$ & 1 planta (9*) Rozas de Puerto Real (S.P.E.F.) \\
\hline $4 \mathrm{~m}:$ & 1 planta (6*) San Martín de Valdeiglesias \\
\hline $4,5 \mathrm{~m}:$ & $\begin{array}{l}1 \text { planta }\left(3^{*}\right) \text { : Ciempozuelos, Cobeña, Puebla de la Sierra, } \\
\text { Torrejón de Velasco, Valdemoro } \\
2 \text { plantas (5*): Montejo de la Sierra, Chinchón }\end{array}$ \\
\hline $5 \mathrm{~m}:$ & $\begin{array}{l}1 \text { planta (1*): Ciempozuelos, Cobeña, Puebla de la Sierra, } \\
\text { Torrejón de Velasco, Valdemoro }\end{array}$ \\
\hline $6 \mathrm{~m}:$ & $\begin{array}{l}1 \text { planta (2*): Ciempozuelos, Cobeña, Puebla de la Sierra, } \\
\text { Torrejón de Velasco, Valdemoro } \\
2 \text { plantas: Colınenar de Oreja }\end{array}$ \\
\hline $6,5 \mathrm{~m}:$ & $\begin{array}{l}2 \text { plantas }\left(9^{*}\right) \text { : Rozas de Puerto Real (S.P.E.D.) } \\
2 \text { plantas }\left(10^{*}\right) \text { : Estremera }\end{array}$ \\
\hline $7 \mathrm{~m}:$ & $\begin{array}{l}2 \text { plantus: Chinchón } \\
1 \text { planta }\left(3^{*}\right) \text { : Torrelaguna } \\
2 \text { plantas }\left(5^{*}\right): \text { Redueña, Vaddetorres del Jarama, } \\
\text { Villanueva de la Cañada }\end{array}$ \\
\hline 7,5 m: & 2 plantas (5*): Montejo de lat Sierra \\
\hline $8 \mathrm{~m}:$ & I planta $(1 *)$ : Torrelaguna \\
\hline $9 \mathrm{~m}:$ & $\begin{array}{l}\text { Brea del Tajo }\left(2^{*}\right) \\
1 \text { planta }\left(2^{*}\right): \text { Torrelaguna } \\
3 \text { plantas }\left(7^{*}\right) \text { : Colmenar de Oreja }\end{array}$ \\
\hline $10 \mathrm{~m}:$ & $\begin{array}{l}2 \text { plantas (4*): Redueña, Valdetorres del Jarama. } \\
\text { Villanueva de la Cañada }\end{array}$ \\
\hline $10,5 \mathrm{~m}:$ & 2 plantas (4*): Montejo de la Sierra \\
\hline
\end{tabular}

- Entre paréntesis si se especifica para alguna zona del suelo no urbanizable.

Notas sobre medición de alturas:

(1*) Desde el alero a cualquier punto circundante del terreno.

(2*) Desde la cara superior del forjado o solera del edificio hasta la cumbrera o punto más alto.

(3*) Entre la cara superior de la solera y el alero de la cubierta.

(4*) Entre la rasante media del terreno y cumbrera o punto más alto.

(5*) Medidos desde la rasante media del terreno y la comisa, alero y tirante en construcciones de estruntura de cerchas en cubierta. Dicha medida se respetará aun cuando el tirante sea peraltado. En cualquier caso la cumbrera o punto más alto de la cubierta. sin contar los conductos de ventilación o chimeneas. no superará los tres metros medidos a partir del plano horizontal definido en la condición anterior.

(6*) Altura de la cornisa desde la rasante oficial o cota del terreno.

(7*) Excepcionalmente cuando se trate de edificaciones de interés público y social y se justifique la necesidad de alcanzar esta altura por razones de tipo funcional.

(8*) Distancia entre la rasante oficial, o en su defecto, la cota del terreno en contacto con la edificación en su punto más bajo, y la linea de remate de la cubierta o de la edificación principal, caso de ser aquélla plana. (N.S.P.M. de Villanueva de la Cañada 1987).

(9*) La altura se mide en la vertical que pasa por el punto medio de la alineación oficial exterior en cada solar, y desde la rasante oficial de acera hasta el plano inferior del forjado de la última planta en la alineación de fachada. (N.S.P.M. de Rozas de Puerto Real 1984).

(10*) La altura se mide por la distancia vertical desde la rasante de la acera o del terreno. en su caso, en contacto con la edificación a la cara inferior del forjado horizontal que forma el techo de la última planta. (N.S.P.M. de Estremera 1988). 
Tabla 9.- Municipios que en sus normas subsidiarias especifican en suelo no urbanizable la inclización de las cubiertas.

\begin{tabular}{|c|c|}
\hline \multicolumn{2}{|c|}{ PRIMER GRUPO } \\
\hline Prohiben cubierta plana: & $\begin{array}{l}\text { Ciempozuelos } \\
\text { Cobeña } \\
\text { Puebla de la Sierrá } \\
\text { Torrejón de Velasco } \\
\text { Valdemoro (Sólo para las construcciones } \\
\text { cuyo carácter y tipología lo aconseje) }\end{array}$ \\
\hline \multicolumn{2}{|c|}{ SEGUNDO GRUPO } \\
\hline Fijan un máximo de inclinación. Máximo de $30^{\circ}$ : & $\begin{array}{l}\text { Estremera } \\
\text { Horcajo de la Sierra } \\
\text { Montejo de la Sierra } \\
\text { Navalcarnero } \\
\text { Redueña } \\
\text { Rozas de Puerto Real } \\
\text { Torrejón de Velasco } \\
\text { Valdemoro } \\
\text { Valdetorres del Jarama } \\
\text { Villanueva de la Cañada }\end{array}$ \\
\hline \multicolumn{2}{|c|}{ TFRCER GRUPO } \\
\hline Ponen un intervalo de inclinación entre $15^{\circ}-30^{\circ}$ : & $\begin{array}{l}\text { Batres } \\
\text { Ciempozuelos } \\
\text { San Martin de Valdeiglesias }\end{array}$ \\
\hline
\end{tabular}

Tabla 10.- Municipios que en sus N.S.P.M. especifican en suelo no urbanizable la utilización del color en la cubierta.

\begin{tabular}{|c|c|}
\hline \multicolumn{2}{|c|}{ RFCOMIILNDAN O PERMITEN } \\
\hline Teja color rojo o similar: & $\begin{array}{l}\text { Ciempozuelos } \\
\text { Torrejón de Velasco }\end{array}$ \\
\hline Materiales de color rojo: & San Martin de Valdeiglesias \\
\hline Fibrocemento de colores terrosos u ocres: & $\begin{array}{l}\text { Montejo de la Sierra } \\
\text { Navalcarnero } \\
\text { Redueña } \\
\text { Valdetorres del Jarama } \\
\text { Villanueva de la Cañada }\end{array}$ \\
\hline \multicolumn{2}{|c|}{ PROIIIBEN } \\
\hline Fibrocemento: & $\begin{array}{l}\text { Chinchón } \\
\text { Estremera } \\
\text { San Martin de Valdeiglesias }\end{array}$ \\
\hline
\end{tabular}

Tabla 11.- Municipios que en sus N.S.P.M. especifican en suelo no urbanizable la utilización del color en la fachada.

\begin{tabular}{|l|l|}
\hline \multicolumn{2}{|c|}{ RECOMIENDAN O PERMITEN } \\
\hline Revocos blancos: & Ciempozuelos \\
\hline $\begin{array}{l}\text { Acahados pintados en colores blancos u ocres (tierras) claros } \\
\text { a excepción de los zócalos: }\end{array}$ & Estremera \\
\hline $\begin{array}{l}\text { Revocos en tonos claros y dentro de la gaana comprendida } \\
\text { entre el ocre y el tierra siena tostado: }\end{array}$ & Ciempozuelos \\
\hline Enfoscado colores arena: & Buitrago \\
\hline Pintados en colores tierra y ocres: & $\begin{array}{l}\text { Montejo de la Sierra } \\
\text { Navalcarnero } \\
\text { Redueñá } \\
\text { Valdetorres del Jarama } \\
\text { Villanueva de la Cañada }\end{array}$ \\
\hline Ladrillo pardo de uso local: & Batres \\
\hline Lidrillo visto cerámico rojizo u ocre: & Ciempozuelos \\
\hline & Buitrago \\
\hline Color blanco: & Ciempozuelos \\
\hline Lidrillo blanco veteado o vidriado: & Chinchón \\
\hline Revoco color gris: & Estretnera \\
\hline Bloque de hormigón gris: & Estremera \\
\hline Plaquetas de revestimientos bituminoso en su color negro: & \\
\hline
\end{tabular}

(c) Consejo Superior de Investigaciones Científicas Licencia Creative Commons 3.0 España (by-nc)

\section{BIBLIOGRAFIA}

COMUNIDAD DE MADRID. 1984 a. Urbanizaciones ilegales. Catálogo. Centro de Información y documentación de la Consejería de Ordenación del Territorio, Medio Ambiente y Vivienda. Madrid.

COMUNIDAD DE MADRID. 1984 b. Urbanizaciones ilegales. Programa de actuación. Centro de Información y documentación de la Consejería de Ordenación del Territorio, Medio Ambiente y Vivienda. Madrid.

CONSEJERÍA DE OBRAS PÚBLICAS Y TRANSPORTE. 1989. Recomendaciones de diseño y adecuación paisajística del entorno, afectado por obras públicas de la Comunidad de Madrid. Oficina de planeamiento territorial. Madrid.

COPLACO. 1975 a. Normas Complementarias $y$ Subsidiarias de Planeamiento del término municipal de El Atazar. Comisión de Planeamiento y Coordinación del área metropolitana de Madrid. Ministerio de Obras Públicas y Urbanismo. Madrid.

COPLACO. 1975 b. Normas Complementarias $y$ Subsidiarias de Planeamiento del término municipal de Titulcia. Comisión de Planeamiento y Coordinación del área metropolitana de Madrid. Ministerio de Obras Públicas y Urbanismo. Madrid.

COPLACO. 1975 c. Normas Complementarias y Subsidiarias de Planeamiento del término municipal de Valdepiélagos. Comisión de Planeamiento y Coordinación del área metropolitana de Madrid. Ministerio de Obras Públicas y Urbanismo. Madrid.

COPLACO. 1976 a. Normas Complementarias y Subsidiarias de Planeamiento del término municipal de Belmonte del Tajo. Comisión de Planeamiento y Coordinación del área metropolitana de Madrid. Ministerio de Obras Públicas y Urbanismo. Madrid.

COPLACO. 1976 b. Normas Complementarias y Subsidiarias de Planeamiento del término municipal de Campo Real. Comisión de Planeamiento y Coordinación del área metropolitana de Madrid. Ministerio de Obras Públicas y Urbanismo. Madrid.

COPLACO. 1976 c. Normas Complementarias y Subsidiarias de Planeamiento del término municipal de El Berrueco. Comisión de Planeamiento y Coordinación del área metropolitana de Madrid. Ministerio de Obras Públicas y Urbanismo. Madrid. 
COPLACO. 1976 d. Normas Complementarias y Subsidiarias de Planeamiento del término municipal de Fresno del Torote. Comisión de Planeamiento y Coordinación del área metropolitana de Madrid. Ministerio de Obras Públicas y Urbanismo. Madrid.

COPLACO. 1976 e. Normas Complementarias y Subsidiarias de Planeamiento del término municipal de Villalbilla. Comisión de Planeamiento y Coordinación del área metropolitana de Madrid. Ministerio de Obras Públicas y Urbanismo. Madrid.

COPLACO. 1976 f. Normas Complementarias y Subsidiarias de Planeamiento del término municipal de Manzanares el Real. Comisión de Planeamiento y Coordinación del área metropolitana de Madrid. Ministerio de Obras Públicas y Urbanismo. Madrid.

COPLACO. 1977 a. Normas Complementarias y Subsidiarias de Planeamiento del término municipal de Santa María de la Alameda. Comisión de Planeamiento y Coordinación del área metropolitana de Madrid. Ministerio de Obras Públicas y Urbanismo. Madrid.

COPLACO. 1977 b. Normas Complementarias y Subsidiarias de Planeamiento del término municipal de Robregordo. Comisión de Planeamiento y Coordinación del área metropolitana de Madrid. Ministerio de Obras Públicas y Urbanismo. Madrid.

COPLACO. 1977 c. Normas Complementarias y Subsidiarias de Planeamiento del término municipal de Somosierra. Comisión de Planeamiento y Coordinación del área metropolitana de Madrid. Ministerio de Obras Públicas y Urbanismo. Madrid.

COPLACO. 1979 a. Proyecto de delimitación de suelo urbano y Normas sobre uso del suelo y edificación para el término de Valdaracete. Comisión de Planeamiento y Coordinación del área metropolitana de Madrid. Ministerio de Obras Públicas y Urbanismo. Madrid.

COPLACO. 1979 b. Proyecto de delimitación de suelo urbano y Normas sobre uso del suelo y edificación para el término de Villavieja de Lozoya. Comisión de Planeamiento y Coordinación del área metropolitana de Madrid. Ministerio de Obras Públicas y Urbanismo. Madrid.

COPLACO. 1979 c. Proyecto de delimitación de suelo urbano y Normas sobre uso del suelo y edificación para el término de La Serna del Monte. Comisión de Planeamiento y Coordinación del área metropolitana de Madrid. Ministerio de Obras Públicas y Urbanismo. Madrid.

COPLACO. 1979 d. Proyecto de delimitación de suelo urbano y Normas sobre uso del suelo y edificación para el término de Gascones. Comisión de Planeamiento y Coordinación del área metropolitana de Madrid. Ministerio de Obras Públicas y Urbanismo. Madrid.

COPLACO. 1979 e. Proyecto de delimitación de suelo urbano y Normas sobre uso del suelo y edificación para el término de Cervera de Buitrago. Comisión de Planeamiento y Coordinación del área metropolitana de Madrid. Ministerio de Obras Públicas y Urbanismo. Madrid.

COPLACO. 1979 f. Proyecto de delimitación de suelo urbano y Normas sobre uso del suelo y edificación para el término de La Hiruela. Comisión de Planeamiento y Coordinación del área metropolitana de Madrid. Ministerio de Obras Públicas y Urbanismo. Madrid.

COPLACO. 1982. Guía para el uso de la cartografia ecológica de la provincia de Madrid. Comisión de Planeamiento y Coordinación del área metropolitana de Madrid. Ministerio de Obras Públicas y Urbanismo. Madrid.

DIRECCIÓN GENERAL DE URBANISMO. 1981. Plan General de Ordenación Urbana del término municipal de Navalcarnero. Consejería de Política Territorial. Comunidad de Madrid. Madrid.

DIRECCIÓN GENERAL DE URBANISMO. 1984. Normas Subsidiarias de Planeamiento del término municipal de Rozas de Puerto Real. Consejería de Política Territorial. Comunidad de Madrid. Madrid.

DIRECCIÓN GENERAL DE URBANISMO. 1985 a. Normas Subsidiarias de Planeamiento del término municipal de Chinchón. Consejería de Política Territorial. Comunidad de Madrid. Madrid.

DIRECCIÓN GENERAL DE URBANISMO. 1985 b. Normas Subsidiarias de Planeamiento del término municipal de San Martín de Valdeiglesias. Consejería de Política Territorial. Comunidad de Madrid. Madrid.

DIRECCIÓN GENERAL DE URBANISMŌ. 1986 a. Normas Subsidiarias de Planeamiento del término 
municipal de Gargantilla de Lozoya. Consejería de Política Territorial. Comunidad de Madrid. Madrid.

DIRECCIÓN GENERAL DE URBANISMO. 1986 b. Normas Subsidiarias de Planeamiento del término municipal de Colmenar de Oreja. Consejería de Política Territorial. Comunidad de Madrid. Madrid.

DIRECCIÓN GENERAL DE URBANISMO. 1986 c. Normas Subsidiarias de Planeamiento del término municipal de Redueña. Consejería de Política Territorial. Comunidad de Madrid. Madrid.

DIRECCIÓN GENERAL DE URBANISMO. 1986 d. Normas Subsidiarias de Planeamiento del término municipal de Horcajo de la Sierra. Consejería de Política Territorial. Comunidad de Madrid. Madrid.

DIRECCIÓN GENERAL DE URBANISMO. 1986 e. Normas Subsidiarias de Planeamiento del término municipal de Gargantilla de Lozoya. Consejería de Política Territorial. Comunidad de Madrid. Madrid.

DIRECCIÓN GENERAL DE URBANISMO. 1987 a. Normas Subsidiarias de Planeamiento del término municipal de Batres. Consejería de Política Territorial. Comunidad de Madrid. Madrid.

DIRECCIÓN GENERAL DE URBANISMO. 1987 b. Normas Subsidiarias de Planeamiento del término municipal de Torrelaguna. Consejería de Política Territorial. Comunidad de Madrid. Madrid.

DIRECCIÓN GENERAL DE URBANISMO. 1987 c. Normas Subsidiarias de Planeamiento del término municipal de Cobeña. Consejería de Política Territorial. Comunidad de Madrid. Madrid.

DIRECCIÓN GENERAL DE URBANISMO. 1987 d. Normas Subsidiarias de Planeamiento del término municipal de Puebla de la Sierra. Consejería de Política Territorial. Comunidad de Madrid. Madrid.

DIRECCIÓN GENERAL DE URBANISMO. 1987 e. Normas Subsidiarias de Planeamiento del término municipal de Brea del Tajo. Consejería de Política Territorial. Comunidad de Madrid. Madrid.

DIRECCIÓN GENERAL DE URBANISMO. $1987 \mathrm{f}$. Normas Subsidiarias de Planeamiento del término municipal de Ciempozuelos. Consejería de Política Territorial. Comunidad de Madrid. Madrid.

DIRECCIÓN GENERAL DE URBANISMO. $1987 \mathrm{~g}$. Normas Subsidiarias de Planeamiento del término municipal de Valdetorres del Jarama. Consejería de Política Territorial. Comunidad de Madrid. Madrid.

DIRECCIÓN GENERAL DE URBANISMO. 1987 h. Normas Subsidiarias de Planeamiento del término municipal de Villanueva de la Cañada. Consejería de Política Territorial. Comunidad de Madrid. Madrid.

DIRECCIÓN GENERAL DE URBANISMO. 1987 i. Normas Subsidiarias de Planeamiento del término municipal de Valdemoro. Consejería de Política Territorial. Comunidad de Madrid. Madrid.

DIRECCIÓN GENERAL DE URBANISMO. 1988. Normas Subsidiarias de Planeamiento del término municipal de Estremera. Consejería de Política Territorial. Comunidad de Madrid. Madrid.

DIRECCIÓN GENERAL DE URBANISMO. 1989. Normas Subsidiarias de Planeamiento del término municipal de Montejo de la Sierra. Consejería de Política Territorial. Comunidad de Madrid. Madrid.

DIRECCIÓN GENERAL DE URBANISMO. 1990 a. Normas Subsidiarias de Planeamiento del término municipal de Torrejón de Velasco. Consejería de Política Territorial. Comunidad de Madrid. Madrid.

DIRECCIÓN GENERAL DE URBANISMO. 1990 b. Normas Subsidiarias de Planeamiento del término municipal de Lozoyuela-las Navas-Siete Iglesias. Consejería de Política Territorial. Comunidad de Madrid. Madrid.

DIRECCIÓN GENERAL DE URBANISMO. 1991 a. Normas Subsidiarias de Planeamiento del término municipal de Cenicientos. Consejería de Política Territorial. Comunidad de Madrid. Madrid.

DIRECCIÓN GENERAL DE URBANISMO. 1991 b. Normas Subsidiarias de Planeamiento del término municipal de Buitrago de Lozoya. Consejería de Política Territorial. Comunidad de Madrid. Madrid.

DIRECCIÓN GENERAL DE URBANISMO. 1991 c. Normas Subsidiarias de Planeamiento del término municipal de Chapinería. Consejería de Política Territorial. Comunidad de Madrid. Madrid.

NAVAJAS, P. 1983 a. Arquitectura vernácula de Madrid. Comunidad de Madrid. Consejería de Ordenación del Territorio, Medio Ambiente y Vivienda. Madrid. 
NAVAJAS, P. 1983 b. Normativa urbanistica y arquitectónica sobre la vivienda rural en la provincia de Madrid. Comunidad de Madrid. Consejería de Ordenación del Territorio, Medio Ambiente y Vivienda. Madrid.

\section{NOTAS}

(1). Ley sobre el Régimen del Suelo y Ordenación Urbana, Texto refundido aprobado por el Real Decreto 1.346/1976 de 9 de abril

(2). Las normas distinguen entre suelo urbano, urbanizable y no urbanizable:

- El suelo urbano comprende las áreas ocupadas por el desenvolvimiento del pueblo y que lo delimitan como tales en los planos de ordenación.

- "Constituyen el suelo apto para urbanizar o urbanizable aquellas áreas del territorio que las N.S.M. destinan a ser soporte del crecimiento previsible" (Dirección General de Urbanismo 1990b).

- Constituyen el suelo no urbanizable aquellos terrenos del término municipal que, por sus valores de orden ecológico, paisajístico, agrario o minero, o por no ser necesarios para usos urbanos, son excluidos del desarrollo urbano por las Normas Subsidiarias de Planeamiento, siendo objeto de medidas tendentes a evitar su degradación y a potenciar y regenerar las condiciones de los aprovechamientos propios del mismo. Los terrenos que lo constituyen se delimitan en el plano de clasificación del suelo del término municipal. (Dirección general de urbanismo 1991c)

"El suelo no urbanizable común está constituido por aquellas áreas en las que no es necesario adoptar especiales medidas de protección. El suelo no urbanizable especialmente protegido es aquél constituido por áreas de destacados valores ecológicos, productivos, paisajísticos o culturales, que deben de ser objeto de una protección específica que tienda a defender y potenciar los valores que han originado su inclusión en esta categoría." (Dirección general de urbanismo 1986e).

(3). Hay que advertir que no todas las normas subsidiarias tienen todos los epígrafes, en algunos casos aparecen clasificados de otra forma, no obstante descle el punto de vista sistemático responden a la clasificación propuesta.
(4). En una posterior revisión, que tenía por objeto ver cuántos del total de los núcleos rurales puros y de las villas que componen la Comunidad de Madrid tenían normas actuales, arrojó el siguiente resultado:

- De 14 municipios rurales puros que contiene la Comunidad Autónoma de Madrid sólo uno, el citado Puebla de la Sierra, tiene editadas las normas actuales.

- De 15 municipios clasificados como villas de la CAM todos tienen editadas las normas actuales excepto uno: Manzanares el Real, que es la única villa que no está clasificada como "conjunto arquitectónico de interés rural".

(5). Se entiende por usos del suelo, a efectos de las Normas, "aquellas utilizaciones del terreno para el desarrollo de actividades concretas que pueden permitirse, según los casos, en las diferentes zonas establecidas" (Dirección General de Urbanismo 1986e)

(6). "Uso principal es aquél de implantación prioritaria en uná determinada zona del territorio. Por tanto se considera mayoritario y podrá servir de referencia en cuanto a la intensidad admisible de otros usos como fracción, relación o porcentaje de él" (Dirección General de Urbanismo 1991a).

(7). "Uso complementario es aquel que puede coexistir con el uso principal sin perder ninguno de ellos las características y efectos que les son propios. Todo esto, sin perjuicio de que su necesaria interrelación obligue a una cierta restricción de la intensidad relativa de los mismos respecto del uso principal" (Dirección General de Urbanismo 1991a).

(8). "Uso prohibido es aquel que por su incompatibilidad por sî mismo o en su relación con el uso principal debe quedar excluido del ámbito que se le señala" (Dirección General de urbanismo 1991a)

(9). Hay que tener en cuenta que para cada tipo de uso se definen diversas clases como son por ejemplo: uso de vivienda, uso de oficina, uso de almacenes, uso industrial etc. De esta forma las normas para cada zona o sector definido en cada municipio (casco urbano, zona de ensanche, zona residencial...) definen los diversos tipos de uso, en el caso más sencillo uso principal, compatible y prohibido; así para una determinada zona o sector, por ejemplo casco antiguo, tendríamos:

- Uso principal: uso vivienda.

- Uso compatible: uso oficina y comercio.

- Uso prohibido: uso industrial.

Dentro de cada una de las clases uso vivienda, oficina, comercio, industrial aparece especificado que tipo de vivien$\mathrm{da}$, oficina, comercio e industria a que se refiere con lo que lo que la complejidad aumenta.

(10). En suelo no urbanizable parece que las normäs se centran en los usos del suelo intentando impedir, sobre todo, la aparición de urbanizaciones ilegales. 